\title{
Ocular manifestations of systemic diseases
}

\section{J. Andonegui}

\section{RESUMEN}

Existe un gran número de enfermedades sistémicas que pueden presentar en algún momento de su evolución manifestaciones oculares. Estas alteraciones pueden provocar sintomatología por sí mismas, pueden ayudar a diagnosticar la enfermedad sistémica que las originó o pueden servir para monitorizar el curso evolutivo de la misma. Se destacan las principales características de una serie de enfermedades sistémicas asociadas a patología ocular que serán estudiadas en detalle a lo largo de esta monografía.

Palabras clave. Enfermedades sistémicas. Manifestaciones oculares.

An. Sist. Sanit. Navar. 2008; 31 (Supl. 3): 7-11.

\begin{abstract}
A great number of systemic diseases can exhibit ocular manifestations during their evolution. These ocular alterations may cause symptoms by themselves, but can also be useful in diagnosing or monitoring the evolution of the systemic condition. The main characteristics of several systemic diseases exhibiting some kind of ocular sign are highlighted. These diseases will be described in detail in this issue.

Key words. Systemic diseases. Ocular manifestations.
\end{abstract}

Servicio de oftalmología. Hospital de Navarra. Pamplona.

\section{Correspondencia:}

José Andonegui Navarro

Servicio de Oftalmología

Hospital de Navarra

Irunlarrea, 3

31008 Pamplona

Tfno. 848422081

E-mail: jandonen@cfnavarra.es 


\section{INTRODUCCIÓN}

El globo ocular puede resultar afectado en infinidad de enfermedades sistémicas de todo tipo. La importancia de esta afectación está en relación con varias circunstancias. En primer lugar, esta extensión de la patología sistémica al globo ocular puede provocar síntomas visuales que como tales requerirán el correspondiente tratamiento. Sirvan como ejemplo la retinopatía diabética, la oftalmopatía tiroidea o las alteraciones retinianas en procesos infecciosos generales. La segunda circunstancia que hemos de considerar es que en ocasiones los signos o síntomas oculares pueden ser la primera manifestación de alguna enfermedad sistémica y su aparición, por tanto, nos puede conducir al diagnóstico y al establecimiento del tratamiento, que si se realiza de forma precoz puede evitar secuelas irreversibles. Dentro de este grupo de situaciones estarían las uveítis asociadas a procesos articulares o a enfermedades sistémicas no infecciosas y, en ocasiones, las metástasis coroideas. También debemos destacar que en ciertos procesos sistémicos, como la enfermedad de Behçet o el Vogt-KoyanagiHarada, la patología ocular puede ser la principal causa de morbilidad. Por último, hemos de tener en cuenta que la retina es el único lugar del cuerpo en el que se puede visualizar de forma directa la microcirculación. Esto resulta fundamental para valorar el grado de afectación sistémica por la hipertensión arterial o para evaluar el riesgo de enfermedad vascular en un paciente.

Estudiar todas o la gran mayoría de las manifestaciones oculares de las enfermedades sistémicas hubiese resultado una tarea ímproba. Para la realización de esta monografía se han elegido una serie de procesos basándonos para ello en factores como su elevada prevalencia, su relevancia o peculiaridad clínica o la importancia de sus adecuados diagnóstico y tratamiento. También se ha incluido un capítulo de toxicidad ocular medicamentosa, dentro del cual se describen las alteraciones provocadas por cuatro grupos de fármacos.

\section{ENFERMEDADES VASCULARES (RETINOPATÍA HIPERTENSIVA)}

La hipertensión arterial es una enfermedad cuya incidencia en los países desarrollados llega a alcanzar el 30\%. Este proceso provoca una serie de cambios característicos en los vasos retinianos ${ }^{1}$ cuyo estudio detallado permitirá valorar la repercusión sistémica de la enfermedad, efectuar su seguimiento y establecer un pronóstico aproximado a medio plazo. Desde el punto de vista oftalmológico las alteraciones de la retinopatía hipertensiva no tienen ningún tratamiento específico. El control adecuado de la tensión arterial es la única alternativa para estos pacientes.

\section{ENFERMEDADES ENDOCRINAS}

\section{Retinopatía diabética}

La prevalencia de retinopatía diabética en la población de los países desarrollados se ha estimado en un $3,4 \%^{2}$. En nuestro entorno, la diabetes es la primera causa de ceguera en las personas en edad laboral. Para prevenir y tratar de forma precoz las alteraciones provocadas en la retina por esta enfermedad, las autoridades recomiendan una revisión anual del fondo de ojo en todos los pacientes diabéticos $^{3}$. A pesar de todos los esfuerzos realizados, hasta la fecha sólo el control intensivo de las cifras de glucemia ${ }^{4}$ y la fotocoagulación con láser ${ }^{5}$ han demostrado ser tratamientos efectivos en esta enfermedad.

\section{Edema macular diabético}

El edema macular es la principal causa de pérdida visual en los pacientes diabéti$\cos ^{6}$. El enfoque de esta patología es un proceso complejo tanto en su diagnóstico como en el tratamiento. Aunque en los últimos años han aparecido varias alternativas terapéuticas para esta enfermedad, tan solo la fotocoagulación con láser ${ }^{7}$ y el control metabólico ${ }^{4}$ han demostrado su eficacia de forma significativa.

\section{Oftalmopatía tiroidea}

La oftalmopatía tiroidea es un cuadro de gran variabilidad en su expresión clíni- 
$\mathrm{ca}^{8}$. Además, con relativa frecuencia las manifestaciones oculares y la tirotoxicosis van disociadas en su evolución ${ }^{9}$. Las alteraciones posibles en esta enfermedad pueden afectar a los párpados, los músculos extraoculares, los tejidos blandos orbitarios, la glándula lagrimal, el nervio óptico, la córnea o la conjuntiva y su abordaje suele resultar complejo.

\section{ENFERMEDADES INFECCIOSAS}

\section{Manifestaciones retinianas en las infecciones sistémicas}

La rica vascularización de la retina y la coroides facilita su colonización hematógena en el transcurso de infecciones sistémicas. Estas infecciones pueden estar producidas por hongos (candidiasis o histoplasmosis), virus (sobre todo del tipo herpes), bacterias (sífilis, tuberculosis o borreliosis) o parásitos (toxoplasmosis o toxocariasis) $^{10}$.

\section{Síndrome de inmunodeficiencia adquirida}

Los pacientes infectados por el virus de la inmunodeficiencia humana pueden presentar múltiples manifestaciones oculares, algunas casi exclusivas de esta enfermedad como es el caso de la retinitis por Citomegalovirus. La aparición de la terapia antirretroviral de gran actividad ha modificado el curso y la expresión clínica de esta enfermedad $^{11}$, pero también ha hecho aparecer nuevas entidades como la uveítis por recuperación inmune ${ }^{12}$.

\section{ENFERMEDADES ARTICULARES}

Entre las enfermedades asociadas al HLA B-27 están la espondilitis anquilosante, el síndrome de Reiter, la artropatía psoriásica y la enfermedad inflamatoria intestinal $^{13}$. Este grupo de enfermedades constituyen una de las causas más frecuentes de uveítis anteriores, que representan en sí una entidad única ${ }^{14}$. Aunque su manejo en general no es complicado, puede llevar a la pérdida grave de la visión si se desarrolla glaucoma o edema macular.

En cuanto a la artritis idiopática juvenil, su complicación más seria es el desa- rrollo de uveítis. Es de carácter crónico, no granulomatosa y recidivante y afecta hasta a un $20 \%$ de los pacientes ${ }^{15}$.

Los pacientes con artritis reumatoide, en el curso de su enfermedad, pueden desarrollar queratoconjuntivitis seca, episcleritis, escleritis y afectación corneal. Esta enfermedad no se suele asociar con uveítis y si ésta aparece, se debe buscar otra etiología como causante ${ }^{16}$.

\section{OTRAS ENFERMEDADES SISTÉMICAS NO INFECCIOSAS ASOCIADAS A UVEÍTIS}

Además de las enfermedades articulares, existen otras enfermedades sistémicas no infecciosas que también se asocian con mucha frecuencia a uveítis. Dentro de este grupo podemos incluir la sarcoidosis, la enfermedad de Behçet, el síndrome de Vogt-Koyanagi-Harada, la esclerosis múltiple, el lupus eritematoso sistémico, la panarteritis nodosa o la granulomatosis de Wegener. En muchos de estos procesos la uveítis puede ser la forma de debut de la enfermedad. Además, en ocasiones es la propia uveítis la principal causa de morbilidad derivada de estas enfermedades ${ }^{17}$, por lo que resulta crucial establecer de forma rápida el diagnóstico e instaurar el correspondiente tratamiento para evitar secuelas oculares graves.

\section{ENFERMEDADES NEUROLÓGICAS (ALTERACIONES OCULARES DE LA ENFERMEDAD CEREBROVASCULAR)}

La enfermedad cerebrovascular puede conducir a la aparición de alteraciones oftalmológicas tales como amaurosis fugax, neuropatía óptica isquémica, alteraciones de los reflejos pupilares, de la motilidad ocular extrínseca o de las vías visuales. Las características de los vasos cerebrales y retinianos son similares y el estudio de los últimos puede ayudar a comprender el ictus y las enfermedades cerebrovasculares relacionadas. De hecho, algunos estudios han puesto de manifiesto la relación existente entre los cambios en la circulación retiniana y la posibilidad de sufrir un ictus ${ }^{18}$. 


\section{ENFERMEDADES NEOPLÁSICAS}

\section{Metástasis coroideas}

Las metástasis coroideas son el tumor intraocular maligno más frecuente. Lo más frecuente es que provengan de carcinomas pulmonares en varones y de carcinomas de mama en mujeres. Pueden ser asintomáticas o asociar pérdida visual u otras alteraciones. Suelen presentar problemas de diagnóstico diferencial, sobre todo con el melanoma amelanótico. Su hallazgo suele implicar un mal pronóstico. El tratamiento estará indicado cuando provoquen pérdida visual, diplopia, dolor o proptosis pronunciada $^{19}$.

\section{Metástasis orbitarias}

También la órbita puede ser el lugar de diseminación metastásica, sobre todo en tumores de mama ${ }^{20}$. Las metástasis orbitarias pueden provocar diplopia, dolor, disminución visual, proptosis y alteraciones de la motilidad ocular ${ }^{21}$. El tratamiento sólo se aplica de forma paliativa cuando la tumoración provoque dolor o pérdida visual.

\section{CÓRNEA Y ENFERMEDADES SISTÉMICAS}

Un número elevadísimo de enfermedades sistémicas presentan en algún momento de su curso evolutivo algún tipo de manifestación corneal. Estas alteraciones pueden aparecer cuando el proceso sistémico está avanzado y su repercusión sería anecdótica, pero también pueden desarrollarse de forma temprana y ayudar por tanto a establecer un diagnóstico precoz. También existe un amplísimo grupo de fármacos cuya ingesta se asocia a yatrogenia corneal $^{22}$.

\section{TOXICIDAD OCULAR MEDICAMENTOSA}

Casi todos los fármacos existentes pueden provocar complicaciones oculares en mayor o menor grado. Entre ellas destacan la retinopatía provocada por el tamoxifeno $^{23}$, la cloroquina ${ }^{24} \mathrm{o}$ la hidroxicloroquina ${ }^{25}$ o el síndrome del iris flácido intraoperatorio, que puede aparecer en pacientes en tratamiento con antagonistas de receptores $\beta 1$-adrenérgicos al ser intervenidos de catarata $^{26}$. También hemos de tener en cuenta que los fármacos con acción anticolinérgica pueden provocar un glaucoma agudo por cierre angular en pacientes predispuestos $^{27}$.

\section{BIBLIOGRAFÍA}

1. HAYREH SS. Classification of hypertensive fundus changes and their order of appearance. Ophthalmologica 1989; 198: 169-260.

2. Kempen JH, O'Colmain BJ, LaSKe MC, HafFner SM, KLEIN R, Moss SE et al. The Eye Diseases Prevalence Research Group. Prevalence of diabetic retinopathy among adults in the United States. Arch of Ophthalmol 2004; 122: 552-563.

3. American Diabetes Association. Standards of medical care for patients with diabetes mellitus. Diabetes Care 2000, 23: S32-S42

4. UK Prospective Diabetes Study Group. Intensive blood-glucose control with sulphonyureas and insulin compared with conventional treatment and risk of complications in patients with type 2 diabetes. Lancet 1998; 352: 837-853.

5. Diabetic Retinopathy Study Research Group: Photocoagulation treatment of proliferative diabetic retinopathy: Clinical application of diabetic retinopathy study (DRS) findings. DRS report number 8 . Ophthalmology 1981: 88: 583-600.

6. Moss SE, KLEIN R, KLEIN BE. The incidence of vision loss in a diabetic population. Ophthalmology 1988; 95: 1340-1348.

7. The Early Treatment Diabetic Retinopathy Study Research Group. Photocoagulation for diabetic macular edema. Early Treatment Diabetic Retinopathy Study report $n^{\circ} 1$. Arch Ophthalmol 1985; 103: 1796-1806.

8. WERNER SC. Ocular manifestations. En: Werner SC, Ingbar SH. The Thyroid. A Fundamental and Clinical Text. New York: Harper \& Row. $3^{\text {a }}$ ed. 1971: 528.

9. Gorman CA. Temporal relationship between onset of Grave's ophtalmopathy and diagnosis of thyrotoxicosis. Mayo Clin Proc 1983; 58: 1515-1519.

10. RodríGuez Ares T, De Rojas Silva M, DíAZ-Llopis M. Enfermedades infecciosas. En: SÁNCHEZ SAlorio M, Díaz-Llopis M, Benítez del CAStillo JM, RodríGuEz AREs MT. Manifestaciones oftalmológicas de las enfermedades generales. 
$77^{\mathrm{a}}$ Ponencia oficial de la Sociedad Española de Oftalmología 2001; 10: 339-375.

11. Goldberg DE, Smithen LM, Angelilli A, FREEMAN WR. HIV-associated retinopathy in the HAART era. Retina 2005; 25: 633-649.

12. JABS DA, van NATTA ML, HolBrook JT, KEMPEN JH, Meinert CL, DAVIS MD. Studies of the Ocular Complications of AIDS Research Group. Longitudinal study of the ocular complications of AIDS: 2. Ocular examination results at enrollment. Ophthalmology 2007; 114: 787-793.

13. Wakefield D, Montanaro A, McCluskey P. Acute anterior uveitis and HLA-B27. Surv Ophthalmol 1991; 36: 223-232.

14. Rothova A, VeenedaAl WG, Linssen A, Glasius E, KiJlstra A, DE JONG PT. Clinical features of acute anterior uveitis. Am J Ophthalmol 1987; 103: 137-145.

15. ZAK M, PEDersen FK. Juvenile chronic arthritis into adulthood: a long-term followup study. Rheumatology 2000; 39: 198-204.

16. Hernández-García C, Collado P, FernándezGuTIÉRREZ B. Manifestaciones oculares de la artritis reumatoide. Queratoconjuntivitis seca. Rev Esp Reumatol 1997; 24: 190-195.

17. Verity DH, Wallace GR, Vaughan RW, STANFORD MR. Behçet's disease: from Hippocrates to the third millennium, $\mathrm{Br} \mathrm{J}$ Ophthalmol 2003; 87: 1175-1183.

18. Mitchell P, Wang JJ, Wong TY, Smith W, KLein R, LEEDER SR. Retinal microvascular signs and risk of stroke and stroke mortality. Neurology 2005; 65: 1005-1009.

19. Amer R, Peter J, Chowers I, Anteby I. Treatment options in the management of choroidal metastases. Ophthalmologica 2004; 218: 372-377.

20. Shields JA, Shields CL, Scartozzi R. Survey of 1264 patients with orbital tumors and simulating lesions. The 2002 Montgomery Lecture, part 1. Ophthalmology 2004; 111: 997-1008.

21. Goldberg RA, Rootman J, Cline RA. Tumors metastatic to the orbit: a changing picture. Surv Ophthalmol 1990; 35: 1-24.

22. Díaz-Llopis M, Benítez del Castillo JM, Belda J, Morillas PJ. Toxicidad ocular por fármacos y drogas. En: SÁNCHEZ SAlORIO M, DíAz-LloPIS M, Benítez del Castillo JM, Rodríguez ARes MT. Manifestaciones oftalmológicas de las enfermedades generales. $77^{\mathrm{a}}$ Ponencia oficial de la Sociedad Española de Oftalmología 2001; 19: 509-518.

23. Alwitry A, GARDNER I. Tamoxifen maculopathy. Arch Ophthalmol. 2002; 120: 1402-1402.

24. Ferreras A, Pinilla I, Abecia E, Pajarín AB, HonRUBia FM. Toxicidad Retiniana Secundaria a Tratamiento con Cloroquina. Archiv Soc Española Oftalmología 2007; 82: 103-108.

25. Franzco M, Rahman A, McLay J. Early Paracentral Visual Field Loss in Patients Taking Hydroxychloroquine. Archiv Ophthalmol 2006; 124: 1729-1733.

26. CHANG DF. Clinical experience with intraoperative floppy-iris syndrome. Results of the 2008 ASCRS member survey. J Cataract Refract Surg. 2008; 34: 1201-1209.

27. KirWAN JF, SuBAK-SHARPE I, TEIMORY M. Bilateral acute angle closure glaucoma after administration of paroxetine. $\mathrm{Br} \mathrm{J}$ Ophthalmol 1997; 81: 252-254. 
